\title{
Ileal Neuroendocrine Tumor G2
}

National Cancer Institute

\section{Source}

National Cancer Institute. Ileal Neuroendocrine Tumor G2. NCI Thesaurus. Code C160440.

A well differentiated, intermediate grade tumor with neuroendocrine differentiation that arises from the ilium. The mitotic count is $2-20$ per $10 \mathrm{HPF}$ and/or the Ki67 index is 3 to 20 percent. 